Meta

Journal des traducteurs

Translators' Journal

\title{
La traduction du nom adjectival en anglais médical
}

\section{François Maniez}

Volume 46, numéro 1, mars 2001

Traduction médicale et documentation / Medical translation and documentation

URI : https://id.erudit.org/iderudit/003548ar

DOI : https://doi.org/10.7202/003548ar

Aller au sommaire du numéro

Éditeur(s)

Les Presses de l'Université de Montréal

ISSN

0026-0452 (imprimé)

1492-1421 (numérique)

Découvrir la revue

Citer cet article

Maniez, F. (2001). La traduction du nom adjectival en anglais médical. Meta, 46(1), 56-67. https://doi.org/10.7202/003548ar

\section{Résumé de l'article}

La traduction du groupe nominal est une tâche délicate pour le traducteur scientifique, en particulier pour celui qui traduit à partir d'une langue comme l'anglais, où la prémodification nominale est d'usage fréquent, vers le français, qui n'a pas recours à cette structure syntaxique. Cet article examine le choix auquel est confronté le traducteur médical devant les structures anglaises contenant des noms adjectivaux (dans un grand nombre de cas, soit le complément de nom soit l'adjectif peuvent être utilisés en français) et étudie quelques-unes des contraintes dont dépend ce choix.
Ce document est protégé par la loi sur le droit d'auteur. L'utilisation des services d'Érudit (y compris la reproduction) est assujettie à sa politique d'utilisation que vous pouvez consulter en ligne.

https://apropos.erudit.org/fr/usagers/politique-dutilisation/ 


\title{
La traduction du nom adjectival en anglais médical
}

\author{
FRANÇOIS MANIEZ \\ Université de Lyon 2 - Lumière, Lyon, France
}

\begin{abstract}
RÉSUMÉ
La traduction du groupe nominal est une tâche délicate pour le traducteur scientifique, en particulier pour celui qui traduit à partir d'une langue comme l'anglais, où la prémodification nominale est d'usage fréquent, vers le français, qui n'a pas recours à cette structure syntaxique. Cet article examine le choix auquel est confronté le traducteur médical devant les structures anglaises contenant des noms adjectivaux (dans un grand nombre de cas, soit le complément de nom soit l'adjectif peuvent être utilisés en français) et étudie quelques-unes des contraintes dont dépend ce choix.
\end{abstract}

\begin{abstract}
Noun clauses pose a particular challenge for translators of scientific documents, especially when translating from English - where the use of nouns as modifiers at the beginning of a noun clause is very common - to French, whose syntax does not allow such a construction. In this article, we look at the choice medical translators are faced with when dealing with English structures that include adjectival nouns (which can either be translated as an adjective or a prepositional clause in most cases) and we try to establish which constraints determine that choice.
\end{abstract}

\section{MOTS-CLÉS / KEYWORDS}

nom adjectival, traduction médicale, corpus parallèle, suffixe adjectival, traduction de l'anglais au français

\section{Introduction}

Dans un ouvrage publié il y a plus d'un quart de siècle, le professeur Sournia (1974) vitupérait l'usage abusif de l'adjectif par certains de ses confrères, regrettant l'ambiguité de formulations telles que "chirurgien infantile» ou "chirurgien cardiaque», voire «blessé grave». Il relevait également un certain nombre d'utilisations de l'adjectif dont la traduction de locutions d'origine anglo-saxonne était responsable, citant entre autres «traitements institutionnels» ou "profils comportementaux». Si ces usages ne soulèvent plus guère d'objections aujourd'hui, le combat contre l'ambiguité que peut générer le calque systématique de la dérivation adjectivale de l'anglais reste plus que jamais justifié. L’adjectif «comportemental» est de fait entré dans la langue française il y a plus de cinquante ans, mais les pourfendeurs d'anglicismes dans la prose scientifique ont l'occasion de se battre actuellement contre d'autres emprunts, tels «développemental» ou "volitionnel», dont l'usage n'est pas encore consacré par les dictionnaires de la langue générale.

Nous n'avons ici mentionné que les cas dans lesquels un néologisme français résulte de l'adoption d'une dérivation adjectivale de l'anglais. Toutefois, la formation de nouveaux adjectifs français sans équivalent anglais est parfois justifiée par un phénomène syntaxique typique des langues germaniques, et de l'anglais en particulier:

Meta, XLVI, 1, 2001 
la prémodification nominale par un nom adjectival. Certes, la traduction par le complément de nom est théoriquement toujours possible, mais l'utilisation de l'adjectif offre plusieurs avantages: d'une part, elle confère à l'expression utilisée une consonance plus technique par son aspect figé; d'autre part, elle évite le choix entre l'article défini ou indéfini pour le complément de nom. Ainsi, fracture reduction sera traduit par «réduction fracturaire», fracture site par «foyer fracturaire», alors que l'adjectif «fracturaire» n'a pas d'équivalent dans le lexique anglais. On peut présumer que de tels termes donnent progressivement naissance à des expressions pour lesquelles l'usage de l'adjectif ne paraît pas indispensable. Ainsi, «risque fracturaire» supplante peu à peu «risque de fracture» comme traduction de fracture risk. Il faut toutefois signaler que l'utilisation de l'adjectif, fustigée par certains comme jargonneuse, permet de gagner en concision dans les cas où la prémodification nominale de l'anglais exprime des relations plus complexes que celles généralement dévolues au complément de nom: c'est le cas de collocations comme «lésion fracturaire» ou «fragment fracturaire», dans lesquelles l'adjectif signifie «résultant d'une fracture, consécutif à une fracture».

La structure du nom adjectival pose donc au traducteur dont le français est la langue cible le problème du choix entre deux structures possibles: celle du complément de nom et celle qui utilisera l'adjectif formé par dérivation à partir du nom français correspondant, si cet adjectif existe. Ce choix est déterminé par l'usage: cell repository est traduit par «banque de cellules», cell wall par "paroi cellulaire», cet usage étant figé dans le cas de ces deux collocations. Toutefois, nous verrons plus loin qu'il est loin d'être arbitraire et dépend au contraire de facteurs syntaxiques ou sémantiques.

Dans cet article, nous nous efforcerons de montrer comment l'utilisation de corpus de langue spécialisée peut contribuer à mettre en lumière certaines caractéristiques des langues de spécialité, et nous tenterons de formuler quelques-uns des principes qui gouvernent le choix du traducteur confronté aux groupes nominaux contenant des noms adjectivaux.

\section{Corpus}

Pour étudier les emplois de l'adjectif dans les deux langues, nous avons eu recours à deux corpus unilingues et à un corpus bilingue aligné.

Le corpus unilingue anglais du cédérom Internal Medicine 1993 (auquel nous ferons référence sous le nom IM93) a été utilisé afin de contrôler la fréquence d'emploi de certains adjectifs anglais par rapport à leurs équivalents français, mais aussi par rapport au nom anglais dont ils sont dérivés. Il s'agit d'un corpus d'extraits de revues médicales de langue anglaise sur cédérom (The Annals of Internal Medicine, British Medical Journal, Journal of the American Medical Association, The Lancet, The New England Journal of Medicine) contenant un module de recherche de mots-clés par la combinaison de chaînes de caractères correspondant à ces mots-clés avec des opérateurs booléens.

La taille en mots d'IM93 est difficile à évaluer, car le cédérom ne contient pas de données statistiques, en dehors d'un dictionnaire donnant la fréquence de chaque mot (sans lemmatisation). Le cédérom contient plus de 9000 textes, dont environ 5000 articles et 4000 lettres aux éditeurs des diverses revues. La taille moyenne des 
articles étant de 2500 mots et celle des correspondances de 250 mots, on peut évaluer la taille du corpus à environ 13,5 millions de mots. Cette évaluation a par ailleurs été confirmée par le comptage des mots sur un échantillon représentant un peu moins de $10 \%$ du corpus.

Nous avons également utilisé un corpus unilingue français (auquel nous ferons référence sous le nom MEDFR) que nous avons nous-mêmes compilé à partir de données médicales recueillies en ligne sur les serveurs des facultés de médecine de diverses universités francophones. Ce corpus, de relativement faible taille par rapport à IM93, a été exploité à l'aide du logiciel de concordance Tact. Il contient un peu plus de 750000 mots.

Le corpus parallèle bilingue a été constitué à partir de 58 articles du Journal of the American Medical Association et de leur traduction. Les articles originaux ont été extraits du corpus IM93 sous la forme de fichiers de type texte. Leurs traductions, qui n'étaient pas disponibles sous forme électronique, ont été encodées à l'aide d'un logiciel de reconnaissance optique de caractère (les traductions sont celles de la version française du Journal of the American Medical Association). L'alignement du corpus a ensuite été effectué par les logiciels Minimark et Multiconcord. Nous ferons référence à ce corpus sous l'appellation MEDTRAD. Il convient de souligner que ce corpus, en raison des difficultés inhérentes à sa compilation et à son alignement, est d'une taille nettement inférieure à celle d'IM93, puisqu'il contient 135000 mots pour la partie anglaise du corpus et 150000 mots pour la partie française. À titre d'exemple, il faudrait, pour que la fréquence observée pour un même mot soit jugée équivalente, que la fréquence d'emploi de ce mot dans IM93 soit près de 100 fois supérieure à celle que l'on observe dans MEDTRAD.

\section{Description des divers types de transposition du nom adjectival}

Nous attribuerons ici au mot transposition le sens donné par Vinay et Darbelnet (1977), sens repris plus tard par Chuquet et Paillard (1987), c'est-à-dire le changement de catégorie grammaticale résultant de l'opération de traduction. Dans le domaine de la langue médicale, les cas de transposition du nom vers l'adjectif dans la traduction de l'anglais vers le français peuvent se diviser en quatre catégories:

a) Le prémodificateur est un nom désignant une partie de l'anatomie ou une fonction de l'organisme.

back pain $\varnothing$ douleur dorsale (dorsalgie)

brain stem $\varnothing$ tronc cérébral

taste buds $\varnothing$ papilles gustatives

sweat gland $\varnothing$ glandes sudoripares

b) Le prémodificateur est un nom désignant une substance présente dans l'organisme. serum iron $\varnothing$ fer sérique

plasma half-life $\varnothing$ demie-vie plasmatique

c) Le prémodificateur est un nom décrivant le type, la forme ou la cause. twin pregnancy $\varnothing$ grossesse gémellaire

sickle cell $\varnothing$ cellule falciforme

heat rash $\varnothing$ érythème calorique

d) Le prémodificateur est un composé à fonction localisante. end-to-side anastomosis $\varnothing$ anastomose termino-latérale blood-brain barrier $\varnothing$ barrière hémato-encéphalique 


\section{Mécanismes favorisant la transposition}

La fréquence de la transposition du nom adjectival vers l'adjectif dans le sens anglais $\varnothing$ français est due à plusieurs phénomènes. Nous distinguerons les contraintes lexicologiques des contraintes syntaxiques.

\subsection{Contraintes lexicologiques}

La tendance à la transposition du nom adjectival provient en partie du fait que certains adjectifs français n'ont pas de dérivation équivalente en anglais. L'examen des suffixes de dérivation les plus productifs en français (-aire, -al, -eux, -ien et surtout -ique) révèle la présence de plus de 400 adjectifs différents dans MEDFR. Bon nombre de ces adjectifs ne peuvent se traduire en anglais qu'à l'aide de la prémodification par un nom adjectival. Pour ne considérer que le cas des dérivations par le suffixe -aire, des adjectifs tels que «canalaire», «fracturaire», «métaphysaire», « ovalaire», "plasmocytaire», "pubertaire», «séquellaire» ou «tissulaire» n’ont pas d'équivalent anglais. Cette lacune lexicologique est encore plus frappante dans le domaine des adjectifs composés, où l'anglais est beaucoup moins productif que le français: les équivalents anglais font défaut pour des adjectifs tels que "cholangiocellulaire", «dorso-lombaire», «endo-biliaire», «fémoro-patellaire» ou «fibroglandulaire».

Dans certains cas, l'adjectif anglais existe mais n'est que rarement utilisé; c'est le cas de plasmatic, dont la fréquence dans IM93 est de 3, alors que le nom plasma compte plus de 4000 utilisations dans ce même corpus. À titre d'exemple, on dénombre dans MEDTRAD 19 traductions de plasma par l'adjectif "plasmatique», alors que la structure avec complément de nom n'est utilisée que trois fois dans la partie française du corpus («échantillons de plasma» pour traduire à deux reprises plasma samples, et "administration de plasma» pour plasma transfusion). Les noms anglais les plus fréquemment employés à la suite de plasma sont par ordre de fréquence level (4), concentration (3), exchange (3) et activity (2), le mot level étant traduit par «niveau », «concentration » ou « dosage».

L'étude détaillée d'un suffixe de dérivation un peu mois productif que ceux que nous avons cités plus haut (-onnel) nous permettra de souligner quelques lacunes de l'anglais dans ce domaine précis. Le Tableau 1 nous montre les fréquences comparées de ces adjectifs et de leurs équivalents anglais dans les deux corpus unilingues. (Rappelons qu'eu égard à la différence de taille entre les deux corpus, une fréquence relative égale se traduirait par une fréquence 100 fois supérieure en anglais). L'examen du tableau 1 met en lumière les faits suivants:

Tableau 1 : Fréquence comparées de quelques adjectifs français en -onnel et de leurs équivalents anglais en -onal dans les deux corpus unilingues

\begin{tabular}{|lrr|lrr|}
\hline ADJECTIF & FR & EN & ADJECTIF & FR & EN \\
\hline attentionnel & 1 & 7 & interventionnel & 40 & 161 \\
confusionnel & 6 & 15 & intra-lésionnel & 2 & 6 \\
constitutionnel & 39 & 205 & jonctionnel & 9 & 44 \\
décisionnel & 6 & 3 & légionnel & 5 & 0 \\
éjectionnel & 6 & 0 & lésionnel & 95 & 6 \\
institutionnel & 3 & 589 & mictionnel & 25 & 0 \\
intermictionnel & 1 & 0 & morpho-constitutionnel & 1 & 0
\end{tabular}




\begin{tabular}{|lrr|lrr|}
\hline ADJECTIF & FR & EN & ADJECTIF & FR & EN \\
\hline morphofonctionnel & 1 & 0 & pressionnel & 1 & 0 \\
motivationnel & 1 & 15 & prétransfusionnel & 2 & 0 \\
obsessionnel & 13 & 29 & réactionnel & 41 & 1 \\
occupationnel & 1 & 921 & rétentionnel & 11 & 0 \\
organisationnel & 1 & 97 & rotationnel & 3 & 19 \\
péri-lésionnel & 21 & 1 & sous-lésionnel & 5 & 0 \\
permictionnel & 5 & 0 & sus-lésionnel & 7 & 0 \\
positionnel & 11 & 55 & tensionnel & 8 & 0 \\
postmictionnel & 8 & 0 & transfusionnel & 15 & 7 \\
post-traductionnel & 2 & 0 & transitionnel & 13 & 82 \\
post-transfusionnel & 2 & 0 & tridimensionnel & 13 & 0 \\
pré-mictionnel & 1 & 0 & volitionnel & 1 & 7 \\
\hline
\end{tabular}

N.B. Certains adjectifs anglais d'emploi rare (tensive, retentive) sont formés à partir de la même base que certains des adjectifs ci-dessus, mais avec un suffixe différent.

a) Un certain nombre d'adjectifs formés à partir du suffixe -onnel n'ont pas d'équivalent anglais en -onal dans IM93. C'est le cas de «rétentionnel», «éjectionnel», «mictionnel», «pressionnel», «tensionnel» et «tridimensionnel». Les adjectifs tensional et tridimensional figurent toutefois dans le dictionnaire unilingue Webster's American Heritage Dictionary.

b) D’autres adjectifs, tels «réactionnel », « décisionnel ", «lésionnel» et "transfusionnel», sont d'un emploi beaucoup plus fréquent que leurs équivalents anglais.

c) Les adjectifs ayant un préfixe à sens prépositionnel n'ont en général pas d'équivalent anglais. Cela est vrai dans les cas où l'adjectif de base n'a lui-même pas d'équivalent anglais (préfixation de «mictionnel» par pré-, per-, inter- ou post- sans équivalent anglais, puisque mictional n'existe pas), mais également dans certains cas où l'adjectif de base fait partie du lexique anglais (transfusional est parfois employé, mais les équivalents des adjectifs «prétransfusionnel» et "post-transfusionnel» sont pretransfusion et posttransfusion, utilisés en tant que prémodificateurs nominaux). On constate la même lacune pour certains adjectifs formés à partir de «lésionnel». (Si perilesional et intralesional sont présents dans IM93, on ne relève pas d'équivalents de «sous-lésionnel» et "sus-lésionnel».) Deux adjectifs composés («morphoconstitutionnel» et «morphofonctionnel») n'ont pas d'équivalent anglais, mais leur fréquence d'emploi en français est trop faible pour permettre une généralisation.

d) Une autre catégorie d'adjectifs peut être décrite comme étant d'un emploi rare dans les deux langues, même si cette rareté est plus marquée en anglais. C'est le cas d'adjectifs comme «attentionnel», «motivationnel» ou «volitionnel». Ces adjectifs, qui ne figurent pas dans la plupart des dictionnaires français, constituent des calques effectués à partir de l'anglais. Il en va de même des adjectifs «interventionnel» et «positionnel» qui, quoique relativement plus fréquents d'emploi dans MEDFR, nous viennent probablement de la littérature médicale anglaise. L'expression «radiologie interventionnelle» est d'usage fréquent, et l'adjectif peut être utilisé pour qualifier d'autres techniques d'examen (échographie, endoscopie). On parle d'anomalies positionnelles en anatomie, de nystagmus ou de vertige positionnel en neurologie, de clonage positionnel en génétique et de clichés positionnels en radiographie. Ces emplois recouvrent ceux qu'on a observés pour positional dans IM93, à l'exception de 
l'expression positional changes, pour laquelle la structure française du complément de nom semble devoir s'imposer.

e) Dans certains cas, la rareté d'emploi des adjectifs français par rapport à leurs équivalents anglais est le symptôme d'une tendance à l'anglicisme dans la traduction d'expressions d'usage fréquent. On parle ainsi de "prise en charge institutionnelle » pour évoquer l'hospitalisation, l'internement ou le passage en maison de retraite (le nom institutionalization est parfois utilisé dans ce sens en anglais). Une «thérapie occupationnelle » fait référence à l'activité professionnelle, comme le prouve l'emploi fréquent de l'adjectif occupational avec des collocants tels que medicine, exposure, illness, disease ou disorder.

Il arrive également qu'une traduction soit consacrée par l'usage, si bien que le type de transposition décrit plus haut explique un déséquilibre entre le nombre d'utilisations d'un adjectif français et de l'adjectif anglais de dérivation correspondante. Ainsi, l'adjectif «systématique» est beaucoup plus employé que son homologue anglais systematic. La traduction quasi automatique du nom adjectival anglais routine par «systématique» (comme dans l'expression routine screening $\varnothing$ dépistage systématique) en est l'explication (cette traduction est d'ailleurs employée lorsque routine qualifie d'autres noms, tels detection, monitoring, treatment, use ou vaccination).

\subsection{Contraintes syntaxiques}

Il est intéressant de noter que l'accumulation des prémodificateurs nominaux fait entrer en jeu un mécanisme de traduction qui doit tenir compte de certaines priorités terminologiques. Ainsi, enzyme levels est souvent traduit par «taux enzymatiques». Toutefois, à l'intérieur du groupe nominal elevations in serum liver enzyme levels, la présence de deux autres prémodificateurs change l'ordre de priorité du mécanisme de transposition, liver enzyme étant généralement traduit par «enzyme hépatique». Le nom anglais serum donnant lui aussi lieu à une transposition vers l'adjectif «sérique» dans la plupart de ses emplois adjectivaux, l'ensemble du groupe nominal est traduit par "élévations des taux sériques des enzymes hépatiques».

Afin de tenter d'établir quelques données chiffrées concernant la pratique de la transposition, nous nous sommes intéressés au cas dans lesquels l'adjectif «hépatique » traduit le nom adjectival liver. L'existence d'un adjectif anglais (hepatic) de même dérivation et d'emploi relativement fréquent nous a permis d'établir un certain nombre de comparaisons.

Nous avons donc créé à partir de notre corpus bilingue deux tables exploitables par un système de gestion de bases de données. La structure de ces deux tables était identique, deux champs de type Caractère étant assignés à la phrase anglaise et à sa traduction française. La première table (hepatic1) comptait 103 enregistrements regroupant l'ensemble des traductions des phrases contenant le mot français «hépatique». La deuxième table (hepatic2) contenait l'ensemble des phrases anglaises contenant l'adjectif hepatic et leur traduction française, soit 26 enregistrements.

\subsection{1. Étude de la table hepatic1}

Dans 77 enregistrements sur 103, l'adjectif hepatic ne figurait pas dans la phrase anglaise originale, le nom adjectival liver donnant lieu à une transposition vers 
l'adjectif dans 59 cas, transposition systématique dans de nombreux cas, comme celui de liver biopsy, traduit par «biopsie hépatique» à six reprises.

L'adjectif hepatic était présent dans 18 phrases de l'original et qualifiait les noms lipase, failure, metastasis, disease, dysfunction, receptor et hemangioma. Parmi tous ces noms, seuls metastasis et disease (les collocants postérieurs les plus fréquents de liver dans notre corpus) sont également présents, précédés du nom adjectival liver. Il est intéressant de noter qu'à l'exception de lipase et receptor, ces noms ont pour point commun de se rapporter à la pathologie. On peut rapprocher cette observation de celle qu'on fait parfois concernant l'alternance d'emploi des couples d'adjectifs «coronaire / coronarien» et «endocrine / endocrinien», le premier adjectif de ces deux paires qualifiant le plus souvent les noms descripteurs de l'anatomie et le deuxième, les noms descripteurs d'une pathologie.

La juxtaposition de deux adjectifs peut donner lieu à certains remaniements. Ainsi, "prélèvements dans le sang veineux hépatique» est utilisé pour traduire hepatic venous sampling, remaniement qui rappelle la redistribution syntaxique observée dans la traduction de sinus venous thrombosis par «thrombose des sinus veineux».

\subsection{2. Étude de la table hepatic2}

La table contient l'ensemble des traductions des phrases contenant le mot anglais hepatic, soit 26 enregistrements. La traduction française comporte l'adjectif «hépatique» dans tous les cas à l'exception de deux phrases: dans l'une, l'adjectif n'est pas traduit car le contexte permet cette ellipse ( $a$ normal hepatic response to hyperestrogenemia $\varnothing$ une réponse normale à l'hyperestrogénie), alors que dans la deuxième, la structure du complément de nom est utilisée en français (the presence or absence of hepatic enlargement $\varnothing$ la présence ou l'absence d'augmentation de la taille du foie). Il semble donc que la présence de l'adjectif anglais induise la présence de l'adjectif français dans la traduction dans la majorité des cas (ici $92 \%$ ).

Il est cependant intéressant de se pencher sur les collocants de liver qui déclenchent le plus souvent la transposition vers l'adjectif. Le tableau 2 en fait le décompte (les noms sont classés par ordre décroissant du pourcentage de transpositions vers l'adjectif dans la traduction française).

On remarquera la grande fréquence d'emploi de la collocation liver disease, ainsi que la variété de ses traductions: hépatopathie (11 occurrences), atteinte hépatique (8), pathologie hépatique (6), maladie hépatique (6). L'utilisation fréquente du nom «hépatopathie» explique le faible pourcentage de transposition observé dans les chiffres du tableau 1, même si l'on peut considérer que liver est alors traduit par la même racine (hepat-) que lorsque l'adjectif est présent dans la traduction. On relève également un emploi du nom hépatologie pour traduire cette même collocation (This approach is difficult in liver disease. $\varnothing$ Cette approche est difficile en hépatologie.). 
Tableau 2: Traduction des collocants postérieurs de liver par l'adjectif «hépatique»

\begin{tabular}{|l|c|c|c|}
\hline Collocants & Fréquence & $\varnothing$ hépatique & $\%$ transposition \\
\hline tumor & 3 & 3 & 100 \\
metastases & 10 & 8 & 80 \\
enzyme & 4 & 3 & 75 \\
dullness & 3 & 2 & 67 \\
disease & 36 & 15 & 42 \\
transplantation & 3 & 1 & 33 \\
biopsy & 7 & 2 & 29 \\
span & 15 & 0 & 27 \\
edge & 28 & 0 & 0 \\
examination & 6 & 0 & 0 \\
size & 5 & 0 & 0 \\
border & 2 & & 0 \\
\hline
\end{tabular}

En ce qui concerne le quatrième collocant de liver par ordre de fréquence (metastasis), il convient de noter que la collocation «métastases hépatiques» est utilisée 11 fois pour traduire liver metastasis et 2 fois pour traduire hepatic metastasis. Cette même collocation est également utilisée à deux reprises pour traduire l'utilisation de metastasis ou de l'adjectif metastatic précédant le groupe prépositionnel to the liver (gastrinoma metastatic to the liver $\varnothing$ les métastases hépatiques des gastrinomes, metastases to the liver $\varnothing$ métastases hépatiques). On voit donc que même dans les environnements où l'adjectif hepatic est utilisé en anglais, la prémodification par liver reste plus fréquente. Par ailleurs, un certain nombre de noms ne sont jamais précédés de l'adjectif hepatic dans le corpus (dome, dullness, edge, enzyme(s), finding, function, mass, nodule, scintiscan, span, tissue, transplant, tumor(s)). Parmi ces noms, edge et span, d'un emploi très fréquent comme le montre le tableau 2, ne donnent presque jamais lieu à une traduction utilisant l'adjectif «hépatique» quand ils sont prémodificateurs de liver.

\subsection{3. Étude de cell employé comme prémodificateur}

Afin de compléter cette étude des contraintes syntaxiques qui pèsent sur le choix des traductions du groupe nominal en langue de spécialité, nous avons relevé dans MEDTRAD les 90 emplois du nom anglais cell en tant que prémodificateur nominal. Dans 39 cas (43\%), l'adjectif cellulaire a été utilisé dans la traduction française de 14 noms anglais distincts (body, communication, cycle, damage, death, differentiation, function, growth, junction, line, membrane, proliferation, surface et wall). Nous nous sommes penchés sur les cas dans lesquels la transposition vers l'adjectif n'avait pas eu lieu. Les groupes nominaux et leurs traductions sont regroupés dans le Tableau 3.

Dans deux cas seulement, le groupe nominal a la forme d'une suite du type cell $N$ : il s'agit des collocations cell repository (banque de cellules) et cell viability (viabilité de la cellule). L'étude des structures syntaxiques des groupes nominaux de forme plus complexe fait apparaitre deux sous-catégories distinctes: celles dans lesquelles le mot cell est précédé d'un adjectif, et celles dans lesquelles il est précédé d'un nom. Les cas dans lesquels cell est précédé d'un adjectif se décomposent de la manière suivante: 
64 MetA, XLVI, 1, 2001

- $\quad$ ADJ cell N (8 occurrences): dans ces groupes nominaux, l'adjectif qualifie toujours cell.

- $\quad \mathrm{ADJ}$ ADJ cell N N (4 occurrences): le deuxième adjectif qualifie toujours cell, et le premier adjectif ne qualifie cell que dans un cas seulement (gastric epithelial cell surface $\varnothing$ surface des cellules épithéliales gastriques).

Tableau 3: Cas de traduction du nom adjectival cell par un complément de nom en français dans le corpus TRADMED

\begin{tabular}{|c|c|c|}
\hline STRUCTURE & GN anglais & GN français \\
\hline ADJ ADJ cell $\mathrm{N}$ & gastric epithelial cell surface & $\begin{array}{l}\text { surface des cellules épithéliales } \\
\text { gastriques }\end{array}$ \\
\hline ADJ ADJ cell $\mathrm{N}$ & perivascular inflammatory cell infiltrate & $\begin{array}{l}\text { infiltrat périvasculaire par les cellules } \\
\text { inflammatoires }\end{array}$ \\
\hline ADJ ADJ cell $\mathrm{N}$ & in vivo colonic cell proliferation & prolifération de cellules coliques in vivo \\
\hline ADJ ADJ cell $\mathrm{N}$ & increased epithelial cell proliferation & $\begin{array}{l}\text { prolifération accrue des cellules } \\
\text { épithéliales }\end{array}$ \\
\hline ADJ ADJ $\mathrm{N}$ cell $\mathrm{N}$ & vascular smooth muscle cell proliferation & $\begin{array}{l}\text { prolifération des cellules musculaires } \\
\text { lisses des vaisseaux }\end{array}$ \\
\hline ADJ cell $\mathrm{N}$ & changes in parietal cell mass & $\begin{array}{l}\text { modifications de la masse des cellules } \\
\text { pariétales }\end{array}$ \\
\hline ADJ cell $\mathrm{N}$ & endothelial cell lysis & lyse des cellules endothéliales \\
\hline ADJ cell $\mathrm{N}$ & epithelial cell membranes & membranes des cellules épithéliales 2 \\
\hline ADJ cell $\mathrm{N}$ & giant cell or granulomatous arteritis & $\begin{array}{l}\text { artérite à cellules géantes ou } \\
\text { granulomateuse }\end{array}$ \\
\hline ADJ cell $\mathrm{N}$ & immune cell activation & l'activation des cellules immunitaires \\
\hline ADJ cell $\mathrm{N}$ & mononuclear cell infiltrate & infiltrat de cellules mononucléées \\
\hline ADJ cell $\mathrm{N}$ & $\begin{array}{l}\text { neuronal, glial, and vascular cell } \\
\text { membranes }\end{array}$ & $\begin{array}{l}\text { membranes des cellules neuronales, } \\
\text { gliales et vasculaires }\end{array}$ \\
\hline ADJ cell $\mathrm{N}$ & $\begin{array}{l}\text { Takayasu's giant cell arteritis } \\
\text { of the coronary arteries }\end{array}$ & $\begin{array}{l}\text { artérite de Takayasu à cellules géantes } \\
\text { des artères coronaires }\end{array}$ \\
\hline ADJ $\mathrm{N}$ cell $\mathrm{N}$ & natural killer cell activity & activité des cellules tueuses naturelles \\
\hline ADJ $N$ cell $N$ & peripheral Schwann cell tumors & $\begin{array}{l}\text { tumeurs des cellules de Schwann } \\
\text { périphériques }\end{array}$ \\
\hline ADJ $\mathrm{N}$ cell $\mathrm{N}$ & killer T-cell activity & activité des cellules $\mathrm{T}$ tueuses (killer) \\
\hline ADJ $\mathrm{N}$ cell $\mathrm{N}$ & free radical-mediated muscle cell injury & $\begin{array}{l}\text { lésions des cellules musculaires médiées } \\
\text { par les radicaux libres }\end{array}$ \\
\hline ADJ $N$ cell $N$ & $\begin{array}{l}\text { pancreatic polypeptide-producing } \\
\text { islet cell tumors }\end{array}$ & $\begin{array}{l}\text { tumeurs des cellules PP produisant des } \\
\text { polypeptides pancréatiques }\end{array}$ \\
\hline cell $\mathrm{N}$ & cell repository and tissue bank & banque de cellules et de tissus \\
\hline cell $\mathrm{N}$ & cell viability & viabilité de la cellule \\
\hline $\mathrm{N}$ cell $\mathrm{N}$ & antibody and T-cell antigen receptors & $\begin{array}{l}\text { récepteurs antigéniques des anticorps et } \\
\text { des cellules } \mathrm{T}\end{array}$ \\
\hline $\mathrm{N}$ cell $\mathrm{N}$ & foam cell formation & formation de cellules spumeuses \\
\hline $\mathrm{N}$ cell $\mathrm{N}$ & Schwann cell growth & croissance des cellules de Schwann \\
\hline $\mathrm{N}$ cell $\mathrm{N}$ & T-cell function & fonction des cellules $\mathrm{T}$ \\
\hline $\mathrm{N}$ cell $\mathrm{N}$ & T-cell unresponsiveness & insensibilité des cellules T \\
\hline
\end{tabular}




\begin{tabular}{|l|l|l|}
\hline STRUCTURE & GN anglais & GN français \\
\hline N cell N & $\begin{array}{l}\text { Walker 256 cell mobility and } \\
\text { chemotaxis in vitro }\end{array}$ & $\begin{array}{l}\text { motricité et chimiotactisme des cellules } \\
\text { Walker 256 in vitro }\end{array}$ \\
\hline N N cell N & breast cancer cell growth & $\begin{array}{l}\text { croissance des cellules tumorales } \\
\text { mammaires }\end{array}$ \\
\hline N N cell N & prostate cancer cell growth & $\begin{array}{l}\text { croissance des cellules de cancer de la } \\
\text { prostate }\end{array}$ \\
\hline N N cell N & prostate cancer cell proliferation & $\begin{array}{l}\text { prolifération des cellules de cancer de } \\
\text { la prostate }\end{array}$ \\
\hline
\end{tabular}

Les cas dans lesquels cell est précédé d'un nom se décomposent de la manière suivante:

- $\quad \mathrm{N}$ cell $\mathrm{N}$ (6 occurrences) : ces exemples concernent principalement des sous-classes de cellules (foam cell, Schwann cell, T-cell, Walker 256 cell).

- ADJ N cell N (4 occurrences) : l'adjectif qualifie cell, sauf dans deux cas faisant intervenir des adjectifs composés de structure ADJ N-participe (free radical-mediated muscle cell injury $\varnothing$ lésions des cellules musculaires médiées par les radicaux libres, et pancreatic polypeptide-producing islet cell tumors $\varnothing$ tumeurs des cellules PP produisant des polypeptides pancréatiques).

- $\quad \mathrm{N} N$ cell $\mathrm{N}$ (3 occurrences): dans ces exemples, le premier nom est un prémodificateur du second. Dans un cas, la traduction utilise deux adjectifs: breast cancer cell growth $\varnothing$ croissance des cellules tumorales mammaires.

- ADJ ADJ N cell N (1 occurrence) : vascular smooth muscle cell proliferation $\varnothing$ prolifération des cellules musculaires lisses des vaisseaux. Il s'agit d'un emploi relativement atypique dans la mesure où l'adjectif smooth qualifie muscle et non pas cell. Toutefois, la traduction consacrée de smooth muscle cells semble bien être «cellules musculaires lisses» et non pas «cellules des muscles lisses». On est là en présence de l'usage d'un adjectif de relation.

Une règle de traduction relativement fiable (dans le cas de cell) semble donc être que l'adjectif «cellulaire» est employé dans presque tous les cas où le nom cell est l'unique prémodificateur du groupe nominal. Dans tous les autres cas de figure, c'est-à-dire lorsque cell est lui-même précédé d'un nom ou d'un adjectif, la traduction par le complément de nom est employée.

On dénombre par ailleurs 21 cas dans lesquels ni «cellule» ni «cellulaire» ne sont utilisés dans la traduction française. Outre les cas d'ellipse rendue possible par le contexte (on a à deux reprises: cell membrane permeability $\varnothing$ perméabilité de la membrane), on relève surtout l'emploi d'équivalents lexicographiquement figés: blood cell $\varnothing$ globule, red blood cell $\varnothing$ érythrocyte, white blood cell $\varnothing$ leucocyte, blood cell count $\varnothing$ numération sanguine.

\section{Conclusion}

Il apparait donc que le choix de la traduction du nom adjectival anglais vers le français dépend d'un grand nombre de facteurs. Les contraintes lexicologiques et syntaxiques que nous avons évoquées plus haut ne sont sans doute pas les seules à entrer en ligne de compte. Nous avons souligné quelques critères sémantiques qui semblent opérer dans certains cas (le couple anatomie / pathologie, par exemple), mais ces observations 
demandent à être confirmées par l'étude d'un plus grand nombre de collocations formées à partir des noms des domaines concernés. Les critères sociolinguistiques ne doivent pas être davantage négligés: là où le spécialiste emploiera «cancer mammaire», il est probable que le profane parlera de cancer du sein.

Quant aux méthodes d'étude du domaine de la traduction du nom adjectival en langue spécialisée, elles doivent certainement s'appuyer à l'avenir sur les outils les plus récents, l'idéal étant sans doute de disposer d'un corpus bilingue aligné de grande taille. Un étiquetage grammatical des deux parties du corpus serait bien entendu une aide précieuse pour la recherche des structures faisant intervenir les noms adjectivaux de grande fréquence (on pense à des vocables tels que heart, lung ou blood). En l'absence d'un tel corpus, l'étude des corpus comparables, comme l'a souligné Teubert (1996), demeure une activité scientifiquement utile, dans la mesure où elle permet d'étudier un usage de la langue qui reste distinct de celui de la langue de traduction, sans que s'attache nécessairement à cette dénomination une nuance péjorative (cf. Baker 1998). Du point de vue de l'étude de ces corpus comparables, le fossé entre la quantité de données disponibles sous forme numérique en anglais et en français, même s'il tend à s'amenuiser, continue de demeurer un problème pour celui qui cherche à constituer un corpus comparable en langue de spécialité.

\section{RÉFÉRENCES}

BAKer, M. (1998): «Réexplorer la langue de traduction: une approche par corpus », Meta, 43-4, p. $480-485$.

BAnks, D. (1998): "Vague Quantification in the Scientific Journal Article," Asp, la revue du GERAS, 19-22, pp. 17-28.

BÉjoint, H. et P. Thoiron (1992): «Macrostructure et microstructure dans un dictionnaire de collocations en langue de spécialité», Terminologie et traduction, 2/3, 1992, p. 513-522.

Bouché, P. (1984): Les mots de la médecine, Paris, Belin.

Chuquet, H. et M. Paillard (1987): Approche linguistique des problèmes de traduction, Paris, Ophrys.

Clear, J. (1996): “Technical Implications of Multilingual Corpus Lexicography," International Journal of Lexicography, 9-3, pp. 265-276.

Guillemin-Flescher, J. (1981): Syntaxe comparée du français et de l'anglais. Problèmes de traduction, Paris, Ophrys.

Hamburger, J. (1982): Introduction au langage de la médecine, Paris, Flammarion.

Heid, U. (1994) : "Relating Lexicon and Corpus: Computational Support for Corpus-Based Lexicon Building in DELIS," Euralex '94: Proceedings of the Sixth Euralex International Congress (MARTin et al., eds), Amsterdam, John Benjamins, pp. 459-471.

Humbley, J. (1998) : «Le terminologue et le spécialiste de domaine», Asp, la revue du GERAS, 1922, p. 137-149.

LAnglois, L. et P. Plamondon (1998) : «Le repérage automatique de collocations équivalentes à partir de bitextes», Euralex '98: Proceedings of the Eighth Euralex International Congress (T. Fontenelle, P. Hiligsmann, A. Michiels, A. Moulin et S. Theissen, dir.), Liège, Université de Liège, p. 175-186.

Leech, G., R. Garside and M. Bryant (1994): "CLAWS4: The tagging of the British National Corpus," Proceedings of the 15th International Conference on Computational Linguistics (COLING 94), Kyoto, s. é., pp. 622-628.

Maniez, F. (à paraitre): "The Use of Electronic Corpora and Lexical Frequency Data in Solving Translation Problems," Lexis in Contrast (B. Altenberg and S. Granger, eds.), Amsterdam and Philadelphia, John Benjamins. 
Maniez, F. (2001): «Extraction d'une phraséologie bilingue en langue de spécialité: corpus parallèles et corpus comparables», Meta, 46-2.

Peters, C. and E. Picchi (1998) : "Bilingual Reference Corpora for Translators and Translation Studies", Unity in Diversity? Current Trends in Translation Studies (L. Bowker, M. Cronin, D. Kenny and J. Pearson, eds), Manchester, St. Jerome Publishing.

Sournia, J.-C. (1974) : Le langage médical moderne, Paris, Hachette.

Teubert, W. (1996) : “Comparable of Parallel Corpora??” International Journal of Lexicography, 93, pp. 238-264.

Van Hoof, H. (1986) : Précis pratique de traduction médicale, Paris, Maloine.

Vinay, J.-P. et J. Darbelnet (1977) : Stylistique comparée du français et de l'anglais, Paris, Didier. 